\title{
Multiplicity of the red supergiant population in the young massive cluster NGC 330^
}

\author{
L. R. Patrick ${ }^{1,2}$, D. J. Lennon ${ }^{1,2}$, C. J. Evans ${ }^{3}$, H. Sana ${ }^{4}$, J. Bodensteiner ${ }^{4}$, N. Britavskiy ${ }^{1,2}$, R. Dorda ${ }^{1,2}$, \\ A. Herrero ${ }^{1,2}$, I. Negueruela ${ }^{5}$, and A. de Koter ${ }^{4,6}$ \\ 1 Instituto de Astrofísica de Canarias, E-38205 La Laguna, Tenerife, Spain e-mail: 1patrick@iac.es \\ 2 Universidad de La Laguna, Dpto. Astrofísica, E-38206 La Laguna, Tenerife, Spain \\ 3 UK Astronomy Technology Centre, Royal Observatory, Blackford Hill, Edinburgh, EH9 3HJ, UK \\ ${ }^{4}$ Institute of astrophysics, KU Leuven, Celestijnlaan 200D, 3001, Leuven, Belgium \\ 5 Departamento de Física Aplicada, Facultad de Ciencias, Universidad de Alicante, Carretera San Vicente s/n, E03690, San Vicent- \\ edel Raspeig, Spain \\ 6 Anton Pannenkoek Institute for Astronomy, Universiteit van Amsterdam, Science Park 904, NL-1098 XH Amsterdam, The Nether- \\ lands
}

Received September 2019

\begin{abstract}
Context. The multiplicity properties of massive stars are one of the important outstanding issues in stellar evolution. Quantifying the binary statistics of all evolutionary phases is essential to paint a complete picture of how and when massive stars interact with their companions, and to determine the consequences of these interactions.

Aims. We investigate the multiplicity of an almost complete census of red supergiant stars (RSGs) in NGC 330, a young massive cluster in the Small Magellanic Cloud.

Methods. Using a combination of multi-epoch HARPS and MUSE spectroscopy, we estimate radial velocities and assess the kinematic and multiplicity properties of 15 RSGs in NGC 330.

Results. Radial velocities are estimated to better than $\pm 100 \mathrm{~m} \mathrm{~s}^{-1}$ for the HARPS data. The line-of-sight velocity dispersion for the cluster is estimated as $\sigma_{1 \mathrm{D}}=3.20_{-0.52}^{+0.69} \mathrm{~km} \mathrm{~s}^{-1}$. When virial equilibrium is assumed, the dynamical mass of the cluster is $\log \left(\mathrm{M}_{\mathrm{dyn}} / \mathrm{M}_{\odot}\right)=5.20 \pm 0.17$, in good agreement with previous upper limits. We detect significant radial velocity variability in our multi-epoch observations and distinguish between variations caused by atmospheric activity and those caused by binarity. The binary fraction of NGC 330 RSGs is estimated by comparisons with simulated observations of systems with a range of input binary fractions. In this way, we account for observational biases and estimate the intrinsic binary fraction for RSGs in NGC 330 as $f_{\mathrm{RSG}}=0.3 \pm 0.1$ for orbital periods in the range $2.3<\log \mathrm{P}$ [days] $<4.3$, with $q>0.1$. Using the distribution of the luminosities of the RSG population, we estimate the age of NGC 330 to be $45 \pm 5$ Myr and estimate a red straggler fraction of $50 \%$.

Conclusions. We estimate the binary fraction of RSGs in NGC 330 and conclude that it appears to be lower than that of mainsequence massive stars, which is expected because interactions between an RSG and a companion are assumed to effectively strip the RSG envelope.
\end{abstract}

Key words. binaries: spectroscopic - stars: late-type - open clusters and associations: individual: NGC 330 - (Galaxies:) Magellanic Clouds

\section{Introduction}

Most massive stars $\left(>8 \mathrm{M}_{\odot}\right)$ reside in binary or higher order multiple systems (e.g. Sana et al. 2013, 2014; Dunstall et al. 2015; Moe \& Di Stefano 2017), and $\sim 70 \%$ interact with a companion during their lifetimes (Sana et al. 2012; Kobulnicky et al. 2014). These interactions have profound effects on the evolution of all stars involved (de Mink et al.2013) and the nature of their subsequent supernova explosions (Podsiadlowski et al. 1992; De Marco \& Izzard 2017).

\section{Send offprint requests to: 1patrick@iac.es}

$\star$ Based on observations collected at the European Organisation for Astronomical Research in the Southern Hemisphere under ESO programmes 60.A-9183, 083.C-0413, 083.D-0549, 084.D-0591, 085.C0614, 085.D-0395, 086.D-0078. Table A.1 is only available in full in electronic form at the CDS via anonymous ftp to cdsarc.u-strasbg.fr (130.79.128.5) or via http://cdsweb.u-strasbg.fr/cgi-bin/qcat?J/A+A/
Red supergiant stars (RSGs) are the evolved products of massive main-sequence (OB-type) stars with initial masses in the range $8<\mathrm{M}<40 \mathrm{M}_{\odot}$ (e.g. Ekström et al. 2012). The most numerous core-collapse supernovae by type are those classified as type II-P, which are thought to arise from stars with initial masses of $8<\mathrm{M}<23 \mathrm{M}_{\odot}$ that explode while in the RSG phase (Smartt 2009, although see Davies \& Beasor (2018) for a reevaluation of the upper mass limit.). The most massive RSGs are thought to evolve back to hotter temperatures and likely explode as blue supergiant stars (e.g. SN1987A, Sonneborn et al. 1987). The RSG phase of evolution therefore is an important factor in the yields of core-collapse supernovae in general (Smartt 2009 2015), and both the binary fraction and multiplicity properties of this evolutionary stage have a strong effect.

As binary systems evolve, the interactions between companions alter not only the evolution of the individual stars, but also affect the measured properties of the population. Stars with close companions merge and rejuvenate, spending more time on the 
main sequence as blue straggler stars (e.g. McCrea 1964; Schneider et al. 2014). When blue straggler stars evolve to the RSG phase, this likely produces a red straggler effect (Britavskiy et al. 2019).

Massive main-sequence stars with companions of intermediate separation and periods (between around 10 up to 1500 days; Podsiadlowski et al. 1992, Sana et al. 2012) interact as the primary evolves off the main sequence and begins to expand dramatically. Binary stellar evolutionary models predict that interacting companions shorten the RSG lifetime by a factor of three at solar metallicities and even more at lower metallicities, resulting in hot massive stars (Eldridge et al. 2008, Götberg et al. 2018), that is, fewer RSGs.

The remaining massive main-sequence stars in binary systems in which the separations of the companions are sufficiently large to prevent significant interaction until at least one companion reaches the RSG phase can in principle be observed through various different methods (e.g. Neugent et al. 2018, 2019; Patrick et al.2019).

Placing observed binary systems in the context of stellar evolution requires careful consideration of the inherent biases of observations and the parameter space over which binary fraction estimates are valid. By measuring radial velocity $(R V)$ variations (or the lack thereof), we are able to estimate the binary fraction and characterise the observed or excluded systems. Given the arguments outlined above, the overall binary fraction of RSGs is expected to be significantly smaller than that of main-sequence massive stars.

To date, relatively few Galactic RSGs are known to reside in binary systems (e.g. VV Cep, Wright 1977). The most easily detectable configuration for an RSG in a binary system is with a B-type companion, and this is indeed mainly what is observed in the Galaxy. To find such systems, Neugent et al. (2018) defined photometric criteria to identify RSGs that are contaminated by blue light from a potential companion. These were recently expanded upon by spectroscopic follow-up of potential binaries in M31, M33, and the Small Magellanic Cloud (SMC) by Neugent et al. (2019), finding that many of the sources with such a blue excess also show spectroscopic evidence of this crowding, which these authors interpret as evidence for binarity. A complementary approach for detecting binarity is via multi-epoch spectroscopy of RSGs. Patrick et al. (2019, hereafter P19) provided the first estimate of the RSG binary fraction $\left(f_{\mathrm{RSG}}\right)$ in the 30 Doradus region of the Large Magellanic Cloud (LMC). For orbital periods in the range $3.3<\log \mathrm{P}$ [days] $<4.3$, they found $f_{\mathrm{RSG}} \sim 0.3$, in good agreement with expectations (Moe \& Di Stefano 2017).

Young massive star clusters are the perfect environment in which to hunt for RSG binaries (Sana et al. 2009, Kiminki \& Kobulnicky 2012). The Local Group of galaxies contains many well-catalogued young massive clusters that contain significant populations of RSGs, for instance, $\mathrm{h}$ - and $\chi$-Persei (Gazak et al. 2014), RSGC01 (Davies et al. 2008), RSGC02 (Davies et al. 2007), and RSGC03 (Clark et al. 2009) in the Galaxy, and NGC 2100 (Patrick et al. 2016) and Hodge 301 (P19) in the LMC. At the lower metallicity of the SMC, the young massive cluster NGC 330 has a well-studied population of RSGs (Arp 1959; Robertson 1974; Feast \& Black 1980) and early-type massive stars (Feast 1972; Grebel et al. 1992, Lennon et al. 1996, 2003; Evans et al. 2006). By targeting such clusters with longbaseline multi-epoch spectroscopic campaigns, we can begin to unveil the binary population of RSGs.

Given the large radii of RSGs, orbital periods shorter than several hundred days for a binary companion cannot be sup- ported (P19). Therefore, hunting for characteristic periodic RV variations is a long-term endeavour as most variations arising from binarity are expected to be of the order of $1-5 \mathrm{~km} \mathrm{~s}^{-1}$, on timescales of several years or more. This amplitude and timescale is also comparable to the variations seen from convective motions in the atmospheres of RSGs, so that care must be given to distinguishing them from genuine binarity.

Red supergiants display a dense forest of stellar absorption features at visible wavelengths that can be useful for highresolution chemical abundance studies (e.g. Cunha et al. 2007), but the blending of these features can be a complicating factor at lower resolution (see Dicenzo \& Levesque 2019, for a recent analysis of atomic line diagnostics). P19 developed a method to exploit this dense forest of absorption features to estimate very precise RVs. This precision, combined with the modest level of atmospheric variability expected in RSGs and the apparent lack of short-period binary systems, makes RSGs ideal kinematic tracers of their local populations.

In this article we study the RSG population of NGC 330 using multi-epoch spectroscopy with the goal of estimating the binary fraction in the cluster. To do this, we employ spectroscopy from two instruments where the data span baselines of more than a year. The RSG sample and observations are described in Section 2. To estimate RVs for our targets, we employ a novel slicing technique that specifically identifies the average atmospheric velocity, making use of the huge number of spectral lines available at optical wavelengths. Our method is outlined in Section 3, and building on previous studies (Patrick et al. 2016, P19), we estimate the kinematic properties and dynamical mass of NGC 330. We concentrate on the multi-epoch measurements in Section 4 where we detail our method of searching for significant variability for each target and estimate the binary fraction of the sample. In Section 5 we estimate the age of NGC 330 based on the distribution of RSG luminosities and summarise the key physical properties of this cluster. We present our main conclusions in Section 6.

\section{Observations}

Spectroscopic observations of RSGs in NGC 330 are from a combination of the Multi Unit Spectroscopic Explorer (MUSE; Bacon et al. 2014) on the Very Large Telescope (VLT) and the High Accuracy Radial velocity Planet Searcher (HARPS, Mayor et al.2003) spectrograph on the ESO 3.6m telescope. The MUSE observations 1 cover the centre of the cluster, and their reduction is detailed in Bodensteiner et al. (2019). Briefly, these data were reduced using the standard ESO MUSE pipeline v2.6. A fraction of faintest pixels within the MUSE field of view is used to estimate the sky spectrum, which is subsequently subtracted from the data. No telluric correction is necessary because the only prominent telluric features are within well-defined wavelength ranges that are taken into account when the RVs are estimated. Source extraction is performed using Hubble Space Telescope (HST) photometry as an input catalogue, after first converting the HST photometry into the MUSE reference frame. On this input catalogue, a tailored point-spread-function fitting routine is used to extract the flux of the sources at each wavelength step.

The MUSE observations span a baseline of over one year between 2017 August and 2018 December, with six separate epochs. Each epoch consists of five exposures of 540 s, with 0 '.7 offsets and a $90^{\circ}$ derotator offset. The observations were conducted in the (wide-field) adaptive optics (AO) mode, providing

\footnotetext{
Proposal ID: 60.A-9183(A)
} 


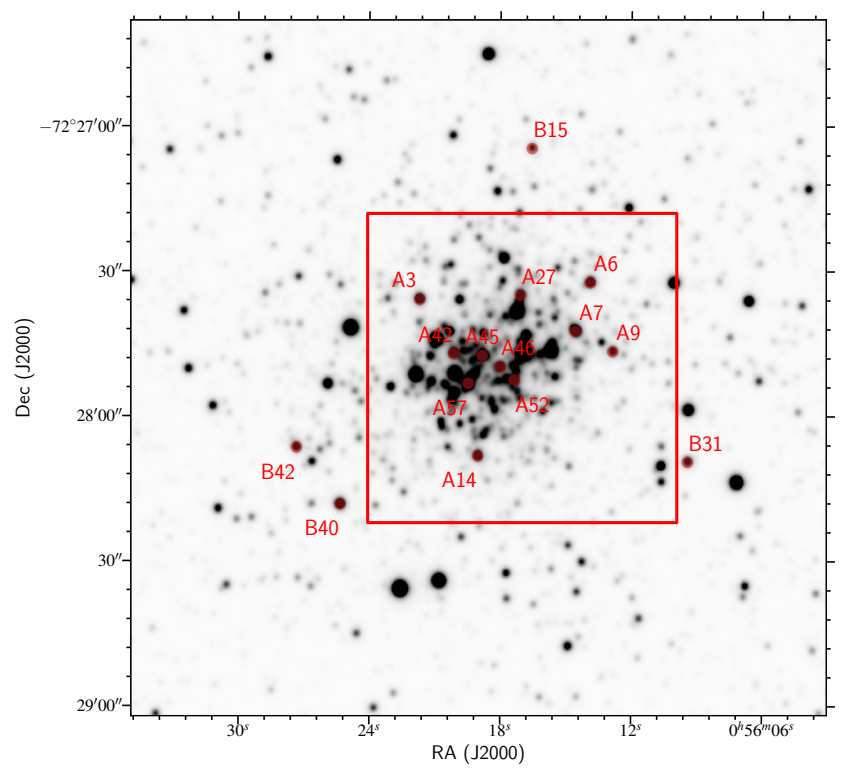

Fig. 1. $B$-band image of NGC 330 obtained with the Wide Field Imager on the 2.2m MPG/ESO telescope (see Momany et al.2001). Red circles indicate our targets, with identifications primarily from Robertson (1974), see Table 1 for details. The approximate footprint of the observed MUSE field (Bodensteiner et al. 2019) is overlaid in red.

spatial sampling of 0 '.2, a wavelength coverage of 4600-9300 and a mean spectral resolving power $(R)$ of $\sim 3000$. The typical signal-to-noise ratios $(\mathrm{S} / \mathrm{N})$ of the RSG spectra are $\sim 25$ around the calcium triplet $(\sim 8500 \AA)$, which is the region used to estimate RVs for the cool star spectra (see Section 3 .

In addition to the recent MUSE observations, focused on the centre of the cluster, we employ archival data from HARPS programmes that targeted cool stars in the Magellanic Clouds? The long-term stability of the HARPS spectrograph is known to be below $1 \mathrm{~m} \mathrm{~s}^{-1}$ (e.g. Dumusque et al. 2012, Pepe et al. 2014) for the highest stability mode (the so-called EGGS mode has a precision of $3-5 \mathrm{~m} \mathrm{~s}^{-1}$, according to the HARPS user manual). The observations used the high-efficiency (wider fibre) socalled EGGS mode, resulting in $R \sim 80000$ over a wavelength coverage of 3800-6900 A. Exposure times range between 1200 and $2400 \mathrm{~s}$ depending on target and programme, which delivers a typical S/N of $\sim 5-15$. The HARPS observations span a oneyear baseline between 2009 October and 2010 November, with a minimum of six observations of each star enabling detection of short-scale variations as well as longer-term trends. HARPS was designed to search for extrasolar planets around cool low-mass stars (Mayor et al. 2003), and it therefore delivers exquisite longterm RV stability and precision, which is ideal for studying the binary fraction among RSGs.

The location of our targets is shown in Figure 11. There are five targets in common between the MUSE and HARPS data: A3, A6, A9, A14, and A42 from Robertson (1974). Given its relative brightness and the interesting results for star B31 from the HARPS data (see Section 4), we also extracted spectra from the wings of the point spread function of this star from the edge of the reduced MUSE datacube. The MUSE footprint also includes an additional six RSGs: Rob74 A7, A27, A45, A46, A52,

\footnotetext{
2 Proposal IDs: 083.C-0413, 083.D-0549, 084.D-0591, 085.C-0614, 085.D-0395, 086.D-0078.
}

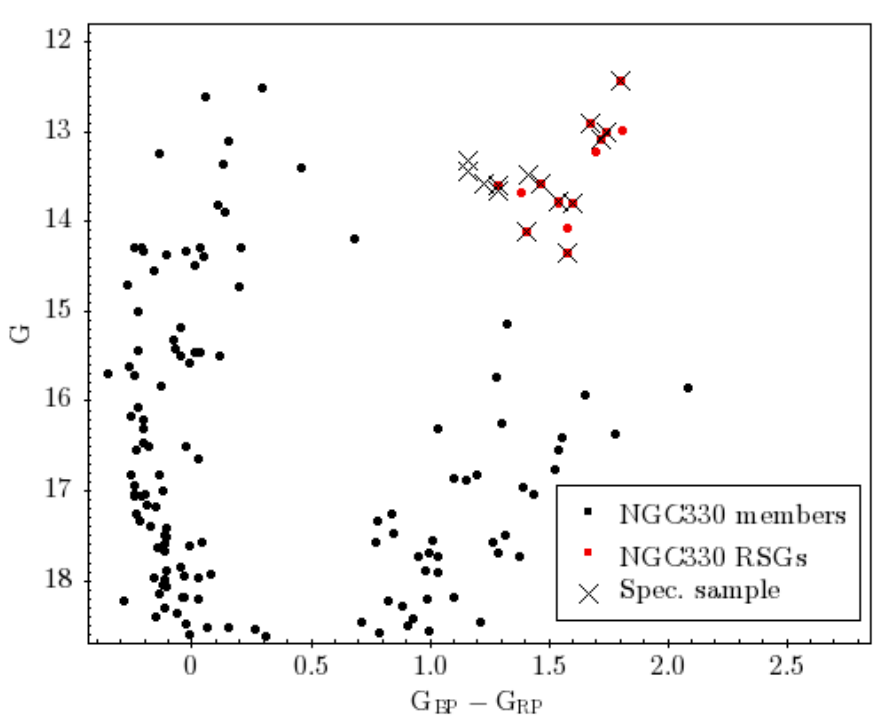

Fig. 2. Gaia $G_{B P}-G_{R P}$ vs. $G$ CMD of the field around NGC 330 . Black points highlight all targets that meet our NGC 330 selection criteria, and red points highlight RSGs selected from the cluster members. Black crosses mark the RSGs with spectroscopic data presented here. This figure highlights the distinction between the RSG populations and lower mass red giant stars as well as blue supergiants in the cluster. Three stars in the spectroscopic sample do not meet the Gaia selection criteria; see text for details.

and A57. The HARPS observations add a further three RSGs: Rob74 B15, B40, and B42.

In total, we present spectroscopic observations for 15 RSGs in NGC 330. Spectral types for our targets were determined from the HARPS and MUSE spectra following the classification criteria and method detailed in Dorda et al. (2018), with the average spectral types for each star listed in Table 1. All targets were cross-matched with Gaia DR2 (Gaia collaboration, Lindegren et al.2018) to assess membership based on proper motions and parallaxes. The Gaia colour-magnitude diagram (CMD) of the cluster is shown in Figure 2, where the potential RSGs in NGC 330 are highlighted in red and the 15 RSGs considered here are also highlighted.

To construct this CMD, we extracted all Gaia sources within 2.5 from the cluster centre (roughly corresponding to the size of Figure 1) and cleaned the sample for parallax and proper motion to select SMC-like candidates. In general, the different populations present in this diagram are in good agreement with those from Carney et al. (1985). We note that a clear gap exists between the RSG population and that of the blue supergiant population at $(B-R)=0-0.2$ and $G>15$, as well as between the RSGs and a population of likely field stars at $(B-R)>0.4$ and $G>16$ (Carney et al. 1985).

All 15 targets in the spectroscopic sample of RSGs have proper motion measurements that are consistent with the bulk movement of the SMC (Gaia Collaboration et al. 2018). Of the 15 targets, 11 have parallax measurements consistent with zero at the two-sigma level. Four targets (A42, A45, A52, and A57) have significant parallax measurements, but the uncertainties are larger than the average. This indicates that these targets are potential Galactic contaminants. However, these four targets are located very near the cluster centre, where potential contamination is more likely as a result of crowding. One target (A46) has a right ascension proper motion measurement that is formally outside our Gaia NGC 330 membership criteria. It is therefore not included as an RSG member in Figure 2, but we consider this 
target a genuine member given that the one-sigma uncertainties on the measurement take it within our proper motion threshold. Based on these data, we conclude that at least 11 of 15 of the targets are genuine SMC members, that is, those with reliable kinematic information from Gaia. The four targets with less reliable Gaia data (A42, A45, A52, and A57) are indicated in Table 1 as potential Galactic contaminants and are included in the sample for further study.

\section{Radial velocities}

The RVs for our targets were estimated using a similar iterative cross-correlation approach as was used by P19. This approach splits the spectra into small wavelength slices that are assumed to provide an independent estimate of the average RV of the star. The slices were compared to a synthetic spectrum extracted from a MARCs stellar model atmosphere from the non-local thermodynamic equilibrium database hosted by the Max Planck Institute for Astronomy ${ }^{3}$. The distribution of RVs estimated from these slices was used to define the RV of the star as well as the uncertainties on this measurement, where a rigorous sigma-clipping approach was used to remove any outlying slices.

To estimate RVs, we used the 6000-6800 $\AA$ range for the HARPS spectra and the 6200-8800 $\AA$ range for the MUSE spectra. These are the reddest parts of the spectra in both cases because this is where the $\mathrm{S} / \mathrm{N}$ is highest. Within these wavelength regions the spectra were split into $42 \AA$ and $167 \AA$ wide wavelength ranges for the HARPS and MUSE spectra, respectively. The synthetic spectrum used to compare to the observation was extracted from an appropriate MARCs model with stellar parameters for an SMC-like RSG (i.e. $\mathrm{T}_{\text {eff }}=4000 \mathrm{~K}, \operatorname{logg}=0.0 \mathrm{dex}$, $[\mathrm{Z}]=-0.75 \mathrm{dex}$ ), and the parameters were convolved by the relevant broadening function for comparison with the observed spectral resolution of the HARPS and MUSE data. The RV for the star is taken to be the mean of the clipped distribution of $\mathrm{RV}$ s from the slices. The uncertainty on this measurement is the standard error on the mean $\left(\sigma / \sqrt{N_{\mathrm{f}}}\right.$, where $N_{\mathrm{f}}$ is the number of retained slices). The typical uncertainties on the HARPS and MUSE data is 0.09 and $1.1 \mathrm{~km} \mathrm{~s}^{-1}$, respectively.

We estimated RVs with the method outlined above because various intrinsic effects perturb the RVs of cool stars as a result of stellar activity in addition to the instrumental effects that can introduce systematic offsets. One of the main sources of uncertainty in estimating RVs for these targets is atmospheric variations. Given the significant atmospheric extension of RSGs, groups of atomic absorption features that are formed at similar depths can display significantly different velocities, with relative differences between groups as large as $25 \mathrm{~km} \mathrm{~s}^{-1}$ (Josselin \& Plez 2007, Kravchenko et al.|2019, although the effect of this on the overall RV estimate is significantly lower). Estimating RVs using only a small number of spectral features can be biased towards atmospheric motions, particularly if these features are dominated by a group of lines at a particular depth.

To attempt to mitigate these effects, we specifically selected the slice width to include a significant number of atomic absorption features. Figure 3 illustrates the absorption features present in a typical slice. A full analysis of the depths at which these features form is beyond the scope of the current study, but we assume that the RV estimate from each slice tends towards the average RV of the star and not the atmospheric variation at a given depth. This is supported by the fact that our sigma-clipping routines remove between 0 and 5 slice measurements from each

\footnotetext{
${ }^{3}$ http://nlte.mpia.de/
}

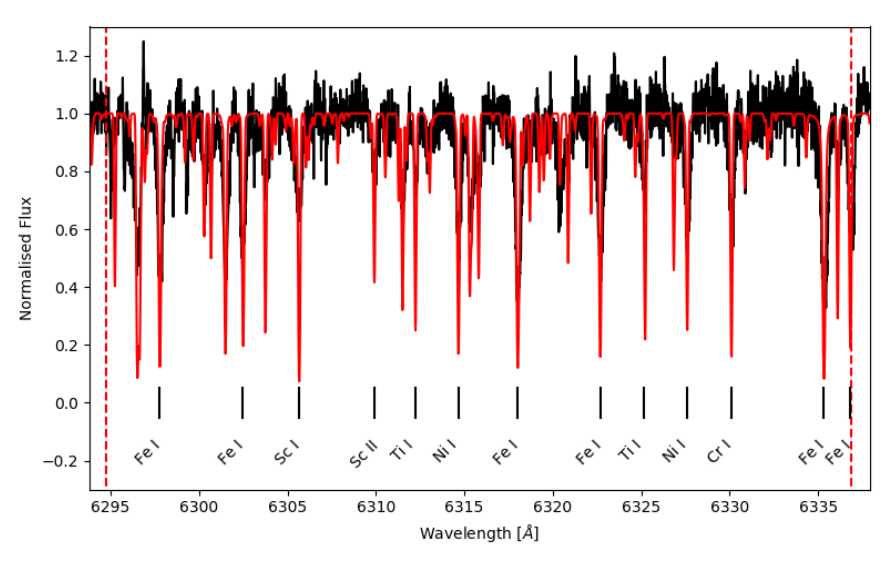

Fig. 3. Example slice used in the RV estimation for the HARPS data. Some of the strongest lines are highlighted, demonstrating the density of spectral features present in a typical slice. The template spectrum calculated from a MARCs model atmosphere is overlaid in red, and the red dashed vertical lines highlight the edges of the slice.

of the final calculations, compared with a total number of 20 slices in the HARPS spectra. Larger variations and accordingly more clipped slices would be expected if atmospheric variability significantly perturbed many slices. In addition, we see no evidence for a wavelength dependence in the RVs estimated from individual slices, but we note that undetected atmospheric variability is the dominant source of uncertainty in the quoted RV measurements on individual epochs.

We also evaluated our choice of synthetic spectrum. To test the extend of the effect of choosing a synthetic spectrum on the precision and stability of the RV estimates, we repeated the analysis using a synthetic spectrum that was extracted with a different radiative transfer code (TURBOSPECTRUM; Plez 2012), with a different line list and stellar parameters. The results of this analysis are that there are no significant differences in the average $\mathrm{RV}$ s for each target. These comparisons reveal a systematic offset of $\sim 0.35 \mathrm{~km} \mathrm{~s}^{-1}$ in the RV estimates of the HARPS data using different synthetic spectra; this difference is undetectable in the MUSE data. This systematic does not affect the internal consistency of the RV estimates, and we therefore conclude that our model is an appropriate choice for these observations, but that on average, our absolute RV cannot be considered more accurate than $\pm 0.35 \mathrm{~km} \mathrm{~s}^{-1}$. We find that in practice, $\mathrm{RV}$ variations that we attribute to atmospheric variation dominate the dispersion of our multi-epoch data, and Table 1 shows that no targets have a standard deviation smaller than $0.5 \mathrm{~km} \mathrm{~s}^{-1}$. However, we note that 11 out of our 15 targets have a dispersion smaller than $2 \mathrm{~km} \mathrm{~s}^{-1}$, which suggests that atmospheric variability has a limited effect on our measurements.

The accuracy of our uncertainties on individual measurements was tested by comparing the results using another welltested method to robustly estimate uncertainties (e.g. Allende Prieto 2007). To do this, we fitted the peak of the crosscorrelation function of the observed spectrum with the model spectrum using a combination of a Gaussian function and a loworder polynomial. The best-fitting model was estimated using a Levenberg-Marquardt algorithm and the least-squares statistic.

When the cross-correlation function of large regions of the spectrum is fitted in the same wavelength regime as was used by the slice analysis, the estimated uncertainties compare reasonably well with those estimated using the slice technique. Typically, the uncertainties estimated using the RV distribution from 
Table 1. Observational information and mean radial velocities $(\bar{v})$ for RSGs in NGC 330. Targets selected by our Gaia criteria without HARPS or MUSE spectra are listed in the No spectra section.

\begin{tabular}{|c|c|c|c|c|c|c|c|}
\hline \multirow[t]{2}{*}{$\overline{\mathrm{ID}}$} & \multicolumn{2}{|c|}{ number of epochs } & \multirow[t]{2}{*}{$\overline{\bar{J}}$} & \multirow[t]{2}{*}{$\overline{\bar{H}}$} & \multirow[t]{2}{*}{$\overline{\bar{K}}$} & \multirow[t]{2}{*}{ SpT } & \multirow{2}{*}{$\begin{array}{c}\bar{v} \pm \sigma \\
\left(\mathrm{km} \mathrm{s}^{-1}\right)\end{array}$} \\
\hline & HARPS & MUSE & & & & & \\
\hline \multicolumn{8}{|c|}{ Spectroscopic sample } \\
\hline A3 & 16 & 6 & 12.019 & 11.469 & 11.374 & G3 Ib & $151.5 \pm 0.5$ \\
\hline A6 & 10 & 6 & 10.969 & 10.217 & 10.087 & K1 Iab & $155.3 \pm 1.1$ \\
\hline A7 & 0 & 6 & 10.193 & 9.408 & 9.182 & G6 Ib & $152.4 \pm 0.9$ \\
\hline A9 & 9 & 6 & 11.778 & 11.072 & 10.874 & $\mathrm{~K} 1 \mathrm{Ib}$ & $154.4 \pm 0.9$ \\
\hline A14 & 9 & 6 & 10.908 & 10.15 & 9.932 & $\mathrm{~K} 1 \mathrm{Ib}$ & $154.6 \pm 1.9$ \\
\hline A27 & 0 & 6 & 11.509 & 10.636 & 10.474 & $\mathrm{~K} 1 \mathrm{Ib}$ & $149.1 \pm 2.4$ \\
\hline $\mathrm{A} 42^{*}$ & 6 & 6 & 11.76 & 11.03 & 10.855 & K0 Ib-II & $153.9 \pm 0.6$ \\
\hline $\mathrm{A} 45^{*}$ & 0 & 6 & 11.592 & 10.916 & 10.719 & G3.5 Ia-Iab & $152.0 \pm 2.2$ \\
\hline A46 & 0 & 6 & 11.643 & 10.952 & 10.653 & $\mathrm{~K} 0 \mathrm{Ib}$ & $157.5 \pm 1.4$ \\
\hline A $52^{*}$ & 0 & 6 & 11.517 & 11.009 & 10.745 & K0 Ib & $162.1 \pm 4.0$ \\
\hline A $57^{*}$ & 0 & 6 & 11.296 & 10.660 & 10.412 & G7 Ia-Iab & $154.6 \pm 1.5$ \\
\hline B15 & 7 & 0 & 12.38 & 11.64 & 11.518 & $\mathrm{~K} 0 \mathrm{Ib}$ & $149.8 \pm 0.6$ \\
\hline B31 & 7 & 1 & 12.233 & 11.491 & 11.339 & K II & $107.8 \pm 14.6$ \\
\hline B40 & 7 & 0 & 10.92 & 10.199 & 10.032 & G7 Iab-Ib & $153.1 \pm 1.1$ \\
\hline B42 & 8 & 0 & 11.842 & 11.158 & 10.991 & G8 Ib-II & $151.9 \pm 0.5$ \\
\hline \multicolumn{8}{|c|}{ No spectra } \\
\hline B10 & 0 & 0 & 10.784 & 10.024 & 9.864 & G8.5 Ia-Iab & $148.0 \pm 4.0$ \\
\hline B19 & 0 & 0 & 11.155 & 10.437 & 10.291 & - & - \\
\hline B20 & 0 & 0 & 11.995 & 11.421 & 11.316 & - & - \\
\hline ARP III-210 & 0 & 0 & 11.934 & 11.226 & 11.084 & - & - \\
\hline ARP III-214 & 0 & 0 & 12.135 & 11.440 & 11.276 & - & - \\
\hline
\end{tabular}

Notes. Literature identification from Robertson (1974. Rob74) and Arp (1959. ARP). See Table B.1 for more information on identification. An asterisk identifies a potential Galactic contaminant based on Gaia data. Spectral type and RV data for B10 are from González-Fernández et al. (2015) because no HARPS or MUSE spectra are available.

the slices are a factor of $1-2$ smaller than the uncertainties estimated by fitting the peak of the cross-correlation function. This adds strength to our uncertainty estimates on individual epochs. However, as noted above, inaccuracies in the synthetic spectrum used and intrinsic atmospheric motions dominate the estimated uncertainties.

To ensure there are no systematic offsets between the MUSE and HARPS results, in the MUSE data the strong telluric absorption features were used to provide an absolute calibration to the RVs, similar to methods used at longer wavelengths (e.g. Lapenna et al. 2015, Patrick et al. 2015, 2017). To do this, a model telluric spectrum was generated using the MOLECFIT tool (Smette et al.|2015). After this correction, we find no significant differences between the HARPS and MUSE spectra. In this analysis, we consider the uncertainties from instrumental stability of the HARPS spectra as negligible in comparison to that of MUSE.

Literature measurements exist for about $50 \%$ of our targets. In general, our results compare well with previous measurements (Feast \& Black 1980, Carney et al.|1985; |Spite et al. 1991; Gonzalez \& Wallerstein 1999, Hill 1999; González-Fernández et al. 2015). See Figure A.1 for a comparison. These comparisons and those in P19 provide us with confidence that this technique provides accurate and precise RV measurements and uncertainties.

\subsection{Kinematic analysis}

Average RVs for each target are listed in Table 1, where the quoted uncertainties are unbiased estimates of the standard de- viation from the multi-epoch observations, taking into account the sample size and measurement errors. Figure 4displays these results (excluding B31, see Section 4) as a function of projected distance from the centre of the cluster (as defined by Mackey \& Gilmore 2003, as $\alpha=00: 56: 18.0, \delta=-72: 27: 47.0, \mathrm{~J} 2000)$. The RVs of all targets are consistent with cluster membership. Additional analysis based on the probability of membership of either NGC 330 or the SMC field was attempted, but given the similarities and overlap between the velocities of these structures, a meaningful comparison was not possible.

Following Patrick et al. (2016), we estimated the line-ofsight velocity and velocity dispersion of NGC 330 using EMCEE (Foreman-Mackey et al. 2013), which is an implementation of the ensembler sampler for the Markov chain Monte Carlo method of Goodman \& Weare (2010). The likelihood function is identical to that of Patrick et al. (2016), where the implicit assumption is that the intrinsic velocity dispersion of the cluster is Gaussian in nature and constant over the radial distance covered by our targets.

After excluding all obviously variable sources (in this case, only B31 was excluded), our estimated line-of-sight velocity for NGC $330\left(\mathrm{v}_{0}\right)$ is $\left(153.7 \pm 1.0 \mathrm{~km} \mathrm{~s}^{-1}\right)$, with a line-of-sight velocity dispersion $\left(\sigma_{1 D}\right)$ of $3.46_{-0.61}^{+0.88} \mathrm{~km} \mathrm{~s}^{-1}$. When we exclude the four potential Galactic contaminants, $\mathrm{v}_{0}$ and $\sigma_{1 D}$ do not change significantly.

NGC 330 also hosts a significant population of B-type stars; RV estimates for seven stars were given by Lennon et al. (2003). $\mathrm{RVs}$ for a larger sample of $\sim 100$ early-type stars in the outskirts and field around the cluster were given by Evans et al. (2006). Figure 4 also includes the RVs for the apparently single OBA- 


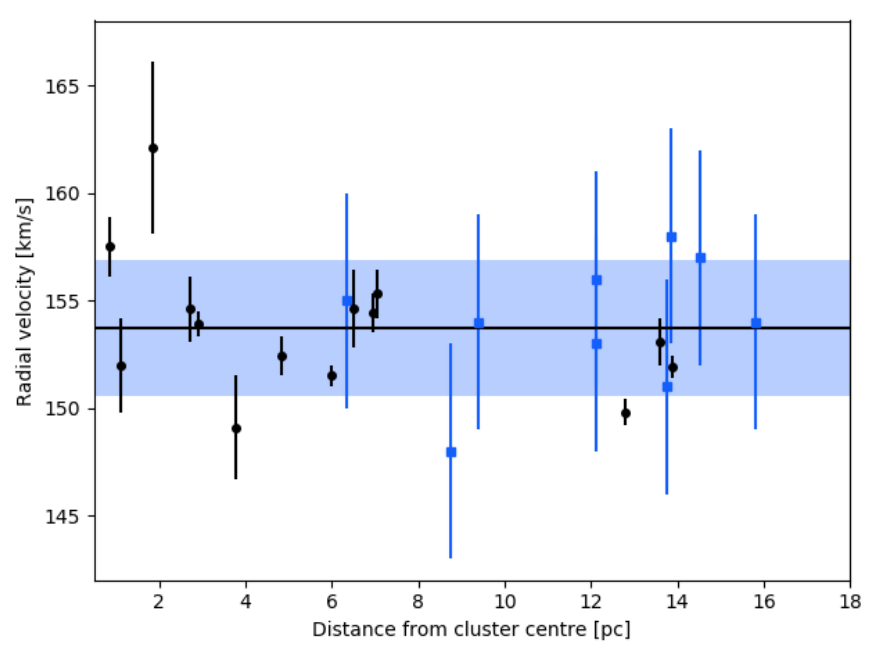

Fig. 4. Average radial velocities as a function of distance from the centre of the cluster (as defined by Mackey \& Gilmore 2003). Black points show average velocities of the RSGs with RV estimates from Table 1 and blue squares show OBA-type stars from Evans et al. (2006). The black solid line and blue shaded region illustrate the systemic velocity and velocity dispersion of the cluster estimated using a combination of RSGs and OBA-type stars $\left(153.7 \pm 3.2 \mathrm{~km} \mathrm{~s}^{-1}\right.$; where the uncertainty quoted and displayed here is the $\sigma_{1 D}$ measured in section 3.1). The black points with the largest uncertainties are those with only MUSE data.

type stars from Evans et al. (2006) that lie within $18 \mathrm{pc}$ from the cluster centre. Notwithstanding the larger uncertainties on the values for the hotter stars, there is generally good agreement between the two samples.

Feast \& Black (1980) estimated $\sigma_{1 D}$ for NGC 330 using both early- and late-type stars, but their results were limited by the precision in their RV measurements. We adopted a similar approach of combining our cool-star RVs with those for early-type stars from Evans et al. Although they potentially trace slightly different populations (Bodensteiner et al.2019) and have larger uncertainties, the OBA stars have similar systemic velocities as the cool stars, and we used them to bolster our sample for the kinematic analysis. After excluding obviously variable OBAtype stars from the sample, we estimate $\mathrm{v}_{0}=153.7 \pm 0.8 \mathrm{~km} \mathrm{~s}^{-1}$ and $\sigma_{1 D}=3.20_{-0.52}^{+0.69} \mathrm{~km} \mathrm{~s}^{-1}$ (in good agreement with the estimate from the RSGs alone). Again, excluding the four potential Galactic contaminants, $\mathrm{v}_{0}$ remains unchanged, whereas $\sigma_{1 D}$ is slightly reduced $\left(2.54_{-0.48}^{+0.63} \mathrm{~km} \mathrm{~s}^{-1}\right)$.

Given their comparative lack of RV variability (e.g. Josselin \& Plez 2007, P19), RSGs are important tracers of the kinematic properties of external young massive clusters. B-type stars (which constitute the majority of the sample from Evans et al. 2006) display intrinsic RV variations within their atmospheres of up to approximately $15 \mathrm{~km} \mathrm{~s}^{-1}$ (Taylor et al. 2014; Dunstall et al. 2015). Despite this and the significantly larger uncertainties on their RVs, including them slightly decreases the uncertainties on $\sigma_{1 D}$. This means that including these targets increases the reliability of the $\sigma_{1 D}$ measurement because the uncertainty on the dispersion is dominated by the number of objects.

\subsection{NGC330 mass estimates}

When we assume the virial theorem, the determination of $\sigma_{1 D}$ allows the dynamical mass $\left(\mathrm{M}_{\mathrm{dyn}}\right)$ of the cluster to be estimated, as follows:

$\mathrm{M}_{\mathrm{dyn}}=\frac{\eta \sigma_{1 D}^{2} r_{\mathrm{eff}}}{G}$

where $r_{\text {eff }}$ is the effective radius and $\eta=6 r_{\text {vir }} / r_{\text {eff }}=11.0$, using the definitions for $r_{\text {vir }}$ and $r_{\text {eff }}$ from Portegies Zwart et al. (2010, and references therein) of 11.14 and 6.11 pc, respectively. The dynamical mass estimated for NGC 330 using the velocity dispersion of RSGs alone is $\log \left(\mathrm{M}_{\mathrm{dyn}} / \mathrm{M}_{\odot}\right)=5.27 \pm 0.20$. A previous estimate of the dynamical mass of NGC 330 is listed by Portegies Zwart et al. (2010) as $\log \left(\mathrm{M}_{\mathrm{dyn}} / \mathrm{M}_{\odot}\right)=5.64$, based on the King profile fits of McLaughlin \& van der Marel (2005).

As shown by P19, RSGs are robust tracers of the dynamical properties of clusters as a result of their small intrinsic $\mathrm{RV}$ variations. In light of the limitations based on the number of RSGs within the cluster, we also estimated the dynamical mass using the OBA-type stars from Evans et al. (2006) within $18 \mathrm{pc}$ of the cluster centre to add strength to the dynamical mass estimate. By combining the two samples, we obtain a dynamical mass estimate of $\log \left(\mathrm{M}_{\mathrm{dyn}} / \mathrm{M}_{\odot}\right)=5.20 \pm 0.17$, again in good agreement with the estimate from RSGs alone. This is significantly larger than the photometric mass estimate of $\log \left(M_{\text {phot }} / M_{\odot}\right)=4.58 \pm 0.2$ from Mackey \& Gilmore (2003) and that of Bodensteiner et al. (2019). This is to some extent expected because $\mathrm{M}_{\mathrm{dyn}}$ measurements made in this way are in general affected by binarity. Gieles et al. (2010) showed that they were able to reproduce the difference in dynamical mass and photometric mass estimates for young clusters such as NGC 330 with a binary fraction among the supergiant population of $25 \%$, which is in good agreement with our binary fraction estimate (presented in Section 4).

\section{4. $R V$ variability and multiplicity analysis}

Long-baseline RV variability studies have been conducted for only a handful of individual Galactic RSGs, for example $\alpha$ Ori (Smith et al. 1989). Josselin \& Plez (2007) studied well-known Galactic RSGs with the goal of identifying atmospheric velocity variability. By calculating RVs of their sample, these authors identified two groups of variability that were split at $\sim 5 \mathrm{~km} \mathrm{~s}^{-1}$, where $\sim 50 \%$ of their sample displayed RV variability above this limit.

P19 studied the RV variation of a sample of 17 RSGs within the 30 Doradus region as part of the VLT-FLAMES Tarantula Survey (VFTS; Evans et al. 2011). The time sampling of the spectroscopy was comparable to our data in NGC 330, and to estimate the RVs, they developed the technique used here (with greater precision possible here due to the high resolution of HARPS). The results from P19 are somewhat at odds with the study of Josselin \& Plez (2007) in the sense that only 1 of 17 sources displayed a RV variation higher than $5 \mathrm{~km} \mathrm{~s}^{-1}$.

The expected level of RV variation for a single RSG as a result of the convective motions within their atmospheres is 1$5 \mathrm{~km} \mathrm{~s}^{-1}$ (Schwarzschild 1975), which has been demonstrated observationally in multiple studies (Spencer Jones 1928; Sanford 1933, Smith et al. 1989; , Josselin \& Plez 2007; Stothers |2010. P19).

The expected level of RV variation for an RSG within a binary system is more difficult to predict. This is because there 
are relatively few literature examples of genuine RSGs within binary systems (e.g. Levesque 2017; Neugent et al. 2019, and fewer still with accurate RV measurements). P19 estimated the expected semi-amplitude variation $(K)$ in the RV for an RSG primary with masses of 8 and $15 \mathrm{M}_{\odot}$ and found that $K$ must be between $\sim 2-30 \mathrm{~km} \mathrm{~s}^{-1}$ with orbital periods greater than $2.5 \mathrm{yr}$ for an $8 \mathrm{M}_{\odot} \mathrm{RSG}$, rising to $4.5 \mathrm{yr}$ for $15 \mathrm{M}_{\odot}$.

Figure 5 shows the RV estimates of our targets from the HARPS and MUSE spectra. Except for B31, all panels show the same relative abscissa. Table A.1 lists the RV estimates for all epochs of each target. The most precise RV measurements are achieved from the HARPS data where the uncertainties reach as low as $\sim 60 \mathrm{~m} \mathrm{~s}^{-1}$, where the precision is, in general, determined by the $\mathrm{S} / \mathrm{N}$ of the spectra.

B31 is a known binary system (Martayan et al. 2007) and will be discussed in more detail by Patrick et al. (in prep). Except for B31, all targets display indications of some low-level variation, with the maximum difference between two epochs in any given target of $4.1 \mathrm{~km} \mathrm{~s}^{-1}$ (A14), in the HARPS data. The general variability in our sample is in good agreement with the levels seen in one half of the sample of Josselin \& Plez (2007), as well as in the highest quality data of P19.

A3 is the best sampled target and displays RV variations with an amplitude of $\sim 1 \mathrm{~km} \mathrm{~s}^{-1}$ in the HARPS data, and with largerthan-average uncertainties, perhaps connected to the fact that it has the earliest spectral type of the sample: it is classified as G3 Ib. There is no detectable trend of RV uncertainty as a function of spectral type. A14, B40, and A42 potentially have signatures of periodic variation in their RV estimates. B15 and B42 have continually increasing RV values over the course of the observational campaign, similar to the trends observed in two stars of Josselin \& Plez (2007). Unfortunately, neither target has follow-up MUSE observations that might constrain their longer term variability.

In the MUSE data, the uncertainties are significantly larger. However, the comparison between the average RVs for targets with both MUSE and HARPS data is generally quite good. For three of the six targets in common, the difference between average RVs is $\sim 1.0 \mathrm{~km} \mathrm{~s}^{-1}$. The three stars outside this range are A3, A14, and B31, all of which are potentially variable. We therefore conclude that the agreement between the HARPS and MUSE data is within the MUSE uncertainties.

\subsection{Multiplicity analysis}

It is important to distinguish, if possible, between RV variability from atmospheric motions (convection, pulsations) and that of variability arising from binary motions. In the case of RSGs, this is difficult to achieve because of the types of binary systems that are expected (Wright 1977) and because of the expected level of variability from their atmospheres (Spencer Jones 1928; Smith et al. 1989; Josselin \& Plez|2007; Stothers 2010; Chiavassa et al. 2010, 2011).

Previous studies that aimed to detect variability owing to binarity where atmospheric variability is a contaminating factor (e.g. Sana et al. 2013; Dunstall et al. 2015, P19, Lohr et al. in prep., Patrick et al. in prep.) used two criteria to detect significant RV variations:

$$
\frac{\left|v_{i}-v_{j}\right|}{\sqrt{\sigma_{i}^{2}+\sigma_{j}^{2}}}>3.0, \text { and }\left|v_{i}-v_{j}\right|>\Delta R V_{\min }
$$

where $v_{i}, \sigma_{i}\left(v_{j}, \sigma_{j}\right)$ are the measured RVs and corresponding uncertainty at epoch $i(j)$ at the epochs $(i$ and $j)$ providing the maximum velocity difference. The first criterion searches for significant variability at the $3 \sigma$ level, taking into account the uncertainties on the measurements. The second criterion $\left(\Delta R V_{\min }\right)$ places a limit on the difference between any two given measurements.

However, given the precision of the HARPS measurements combined with the observed variability as a result of atmospheric variations, the first criterion is always met for these data. Therefore, our variability analysis is based solely on the second variability criterion above. This criterion essentially searches for the largest difference between measured RV measurements, which is an appropriate and effective method for detecting variability, particularly in the regime of sparsely sampled RV curves (e.g. Maoz et al. 2012; Sana et al. 2013; Badenes et al. 2018). Figure 6 illustrates the variation in the number of targets meeting the selection criteria as a function of the $\Delta R V_{\text {min }}$ parameter for HARPS data.

Rather than select a specific value of $\Delta R V_{\min }$ to distinguish between variations from atmospheric motions of single stars and variability from binarity, we chose to evaluate the binary fraction of RSGs in NGC 330 by simulating stellar populations with a range of input binary fractions. The binary simulations used an empirically defined range of parameters from Moe \& Di Stefano (2017) for a flat distribution of orbital periods between $2.3<\log \mathrm{P}$ [days] $<4.3$. The mass-ratio probability density function was defined using a broken power-law distribution defined as $p_{q} \propto q^{-\gamma}$, where $\gamma=-1.7$ for $q>0.3$ and $\gamma=-0.2$ for $0.1<q<0.3$. Primary masses were drawn from a Salpeter initial mass function, over the range $7<\mathrm{M}_{1} / \mathrm{M}_{\odot}<25$, which was chosen to roughly correspond to reproduce the range in masses observed in NGC 330. The eccentricities of the systems were defined based on a power-law distribution probability density function with an exponent of +0.8 in the range $0.0<e<0.8$, and the inclination of the system was drawn at random from angles between 0 and $90^{\circ}$.

Using these parameter distributions, we drew a sample of binary systems with the time sampling, uncertainties, and sample size of the HARPS observations. By solving the positions of each system over time, we estimated the RVs for each epoch, and using the uncertainties, these simulated observations were tested for significant variability using the same criterion as the observations. This process was repeated 10000 times for populations with intrinsic binary fractions in the range from $10 \%$ to $100 \%$. These results are shown alongside the observed distribution in Figure 6, where the shapes of the distributions reflect the sample size.

At small $\Delta R V_{\min }$, the number of significant detections increases to almost $100 \%$. This is a result of the small-scale variations seen in the data and is as expected for a typical RSG. At small $\Delta R V_{\text {min }}$, rather than probing the observed binary fraction, the analysis would therefore be contaminated heavily by atmospheric variations of single RSGs. As $\Delta R V_{\text {min }}$ increases, the number of stars displaying significant variability decreases sharply. By comparing the dashed line in Figure 6, which corresponds to simulations with a binary fraction of $100 \%$, we see that this curve still does not reproduce the abundance of detected variability at small $\Delta R V_{\min }$, nor the sharp drop at around $\Delta R V_{\text {min }}$ $=2-4 \mathrm{~km} \mathrm{~s}^{-1}$. This is further evidence that the variability on this scale is a result of atmospheric velocities arising from single RSGs, in agreement with observations.

To evaluate the intrinsic binary fractions that best reproduce the observed distribution of detected systems as a func- 

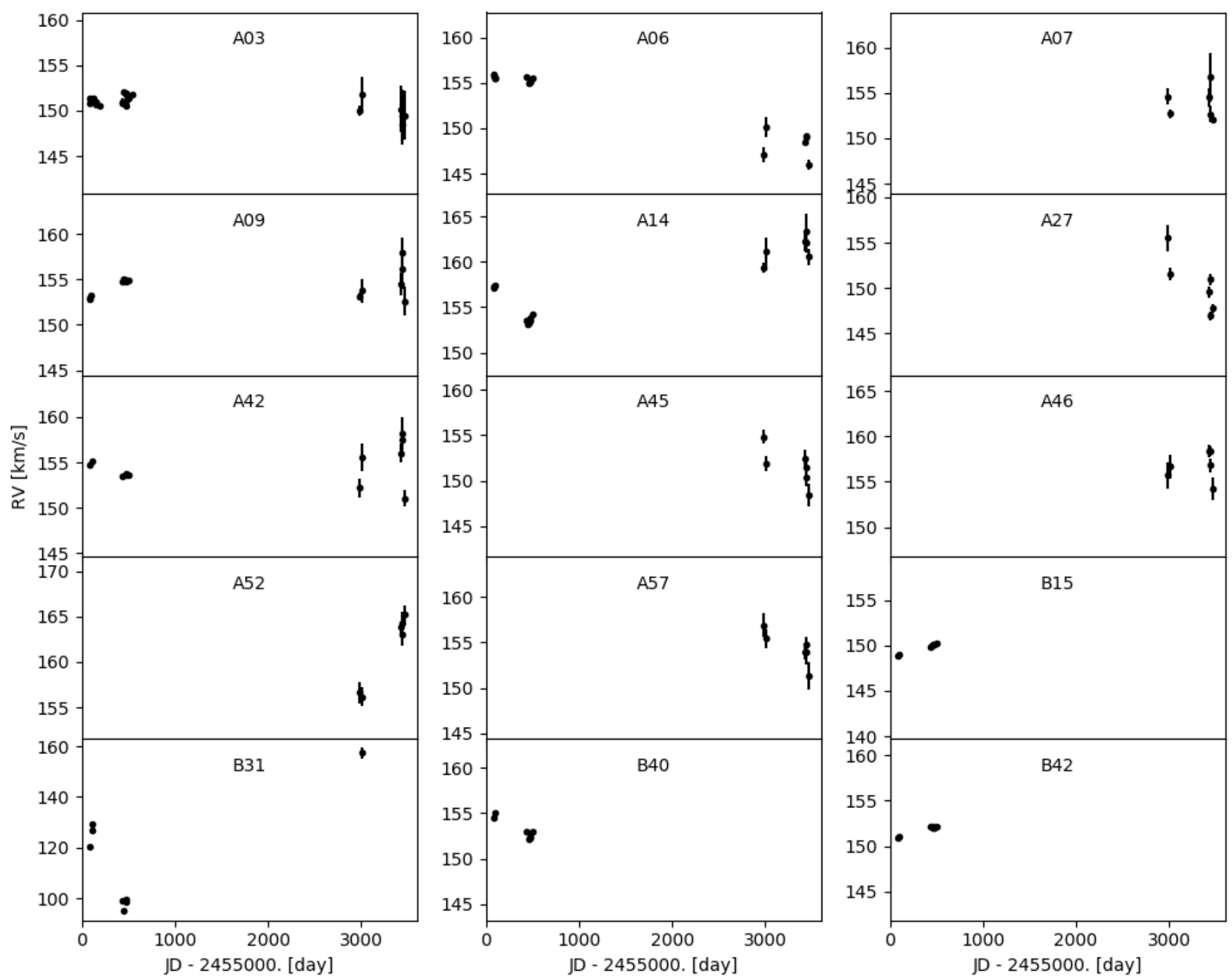

Fig. 5. RV estimates for all targets, shown on the same relative scale to demonstrate the observed differences in RV variations (except for Rob74 B31, which displays significant RV variability; see text for details).

tion of the $\Delta R V_{\min }$ parameter, we ignored the measurements below $\Delta R V_{\text {min }}=4 \mathrm{~km} \mathrm{~s}^{-1}$ because below this value, we assume that atmospheric variations dominate. The goodness of fit was assessed using a $\chi$-squared technique. By averaging the three best-fitting models, we estimate a binary fraction for the RSG population of NGC 300 at $f_{\mathrm{RSG}}=0.3 \pm 0.1$, within the range $2.3<\log \mathrm{P}$ [days] $<4.3, q>0.1$. All binary fractions greater than $30 \%$ over-predict the number of systems detected at large $\Delta R V_{\min }$, where we expect that our observations are reasonably complete.

This estimate is in good agreement with the estimate from P19 in the Tarantula region of the LMC. However, P19 considered a smaller range of periods $(3.3<\log \mathrm{P}$ [days] $<4.3)$ and mass ratios $(q>0.3)$. P19 argued that the size of RSGs excludes orbital periods shorter than $\log \mathrm{P}$ [days] $=2.9$, assuming a radius appropriate for an LMC-like $8 \mathrm{M}_{\odot} \mathrm{RSG}$ (Brott et al. 2011). It is well documented in the literature that the average spectral type of RSGs is dependent upon metallicity (Elias et al. 1985; Massey \& Olsen 2003; Levesque \& Massey 2012; Tabernero et al. 2018), and given the masses implied by the spread of luminosities among the RSG population of NGC 330 (see Section 5.2), we therefore considered orbital periods down to $\sim 200 \mathrm{~d}$. This is sup- ported by the orbital period reported for B31 of $\sim 455 \mathrm{~d}$ (Martayan et al. 2007). The binary fraction of RSGs in NGC 330 does not depend strongly on the range of orbital periods considered. For example, when we extend our analysis to orbital periods in the range $2.3<\log \mathrm{P}$ [days] $<5.3$, we find $f_{\mathrm{RSG}}=0.30 \pm 0.10$. Moe \& Di Stefano (2017) estimated a binary fraction of $\sim 0.3$ for O- and B-type primaries over this range of orbital periods, again in good agreement with the estimate presented here.

\section{Discussion}

\subsection{RSG age of NGC 330}

NGC 330 is a well-observed cluster with several studies of its CMD , implying a range of potential ages from approximately 20 to 40 Myr (see Table 2 and accompanying references). To some extent, this spread in age stems from the difficulty that theoretical isochrones have in simultaneously reproducing salient features in the CMD of this cluster, and in analogous clusters in the Milky Way and the LMC (essentially clusters hosting both BSG and RSG stars). These features include the main-sequence turn-off (MSTO), the main-sequence turn-on (MSTON), or pre- 


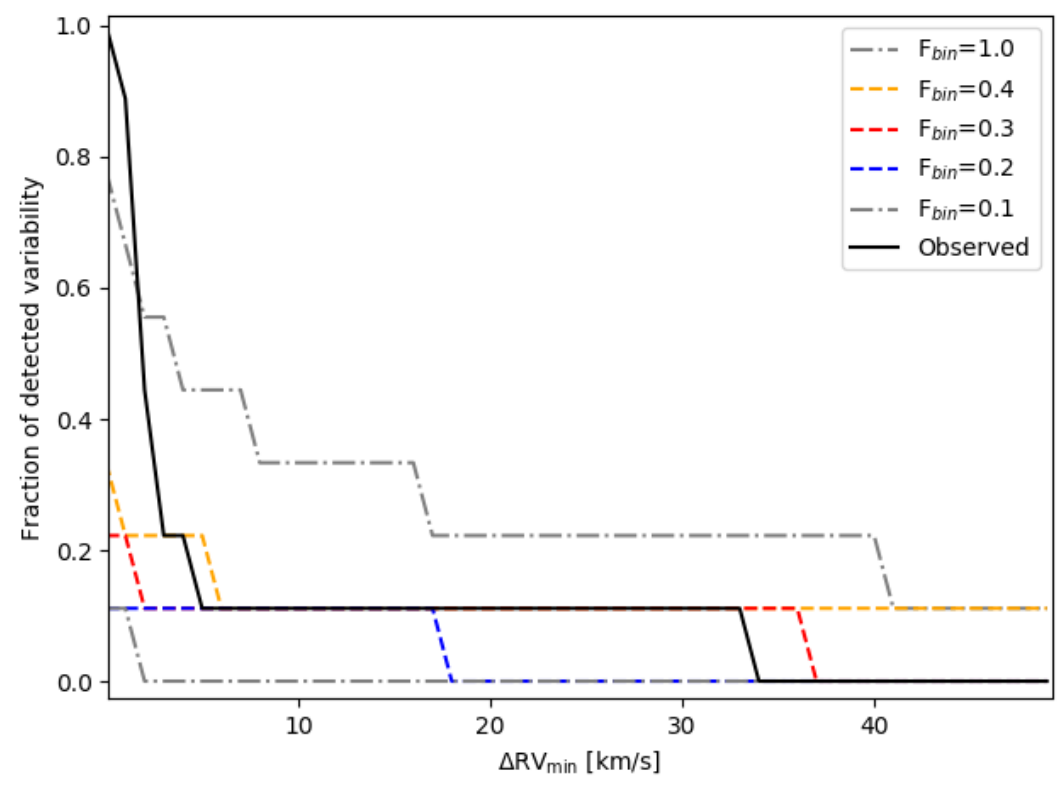

Fig. 6. Observed fraction of detected variability of the RSGs in NGC 330 as a function of the $\triangle \mathrm{RV}_{\min }$ parameter for the nine RSGs with HARPS data (black solid line). The three best-fitting simulated stellar populations with an empirically defined distribution from Moe \& Di Stefano (2017), containing between $20 \%$ and $40 \%$ binary systems with orbital periods in the range $2.3<\log P$ [day] $<4.3$, are shown with dashed coloured lines. The dot-dashed grey lines show the limits of simulations containing a binary fraction of $10 \%$ and $100 \%$. The time sampling, uncertainties, and number of targets of the simulations is chosen to match the HARPS data. Each simulated curve is an average of 10,000 iterations, but to best compare with the observations, the simulated curves are discretised to the same intervals as the observations.

main-sequence stars, the mean magnitude of the BSG stars, and the mean magnitude of the RSG stars (Cignoni et al. 2016). In addition to the above approaches, Britavskiy et al. (2019) and Beasor et al. (2019) have proposed a new technique for determining the ages of such clusters that uses the luminosity distribution of their RSG stars, with the former finding good agreement with the MSTON age of the LMC cluster Hodge 301, but disagreeing with the more traditional MSTO approach. This new technique argues that the luminosity spread of RSGs in a cluster is the result of stellar mergers, recognising that the RSG population of any given cluster is likely affected by multiplicity on the main sequence. In this scenario the most luminous red straggler RSGs are the evolutionary descendants of rejuvenated massive stars on the main sequence that are the result of the merging of a binary system. It then follows that the distribution in luminosity of the faint portion of the RSG branch is an age indicator for a cluster. We used the age-luminosity diagram shown in Figure 7 to estimate the age of NGC 330 with this new method.

For this analysis we selected targets based on their Gaia colours, with the criteria $G<15 \mathrm{mag}$ and $B P-R P>1.2 \mathrm{mag}$ (Gaia collaboration, Lindegren et al. 2018), where the sample was first cleaned based on their kinematic properties, as described in Section 2. This yielded 20 RSGs, 15 of which are from the HARPS + MUSE sample and a further 5 targets outside the MUSE field of view and with no HARPS data. The luminosities of our RSG sample were derived using the $J$-band magnitude calibration from Davies et al. (2013) with a distance modulus of 18.95 (Graczyk et al. 2014).

We estimated the age of NGC 330 using the 10th percentile of the luminosity distribution, resulting in an age of $45 \pm 5 \mathrm{Myr}$ (see Table 2 for a summary of NGC 330 cluster properties). We note that there is a clear distinction (one magnitude in the Gaia $G$ band, see Figure 2 between the least luminous star shown in Figure 7 and the population of less massive red stars in the region. Beasor et al. (2019) demonstrated that the lowest luminosity

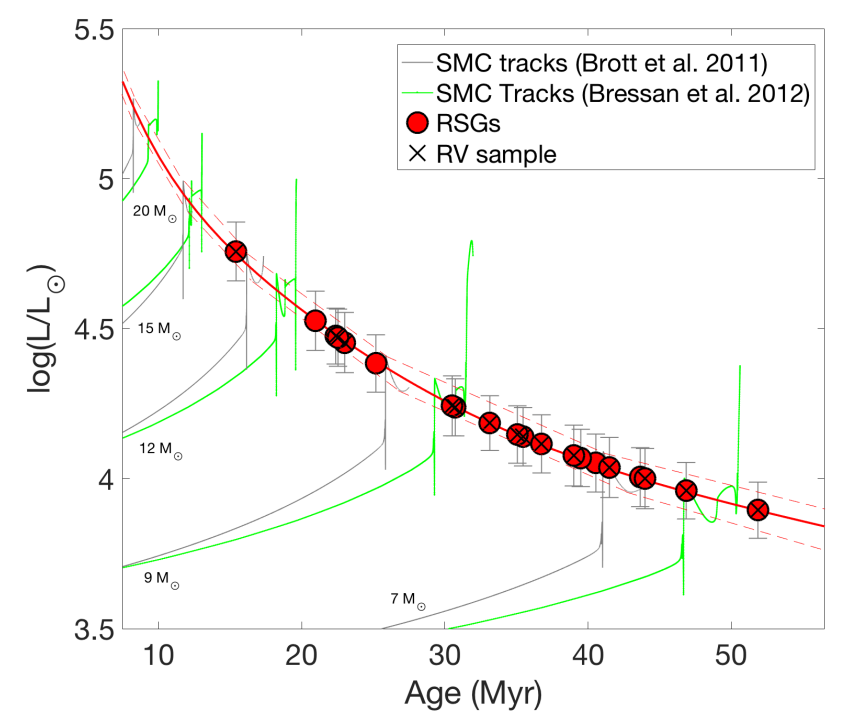

Fig. 7. Luminosity-age diagram for all RSGs in NGC 330, stars that have RV measurements from the present study are marked with a cross. Luminosities are derived the $J$-band magnitude calibration of Davies et al. (2013). Using the least luminous RSGs, we estimate the age of the NGC 330 to be $45 \pm 5$ Myr. SMC-like evolutionary tracks from Brott et al. (2011) are used to estimate ages, and Bressan et al. (2012) tracks are shown to highlight differences between models.

RSG expected for a cluster of 40 Myr containing 50 RSGs with non-rotating MESA isochrones (Dotter 2016) is $\log \left(\mathrm{L} / \mathrm{L}_{\odot}\right)=3.9$, in excellent agreement with what is observed in NGC 330. Table 2 compiles the physical properties of NGC 330 and summarises the main results of this study.

Britavskiy et al. (2019) suggested to use an arbitrary cutoff of $\log \left(\mathrm{L} / \mathrm{L}_{\odot}\right)=4.3$ to distinguish between RSGs and lower- 
Table 2. Physical properties of NGC 330.

\begin{tabular}{llc}
\hline \hline Quantity & Value & Ref. \\
\hline$\tau_{\text {TOFF }}$ & $20 \pm 1.5 \mathrm{Myr}$ & $(1)$ \\
& $25 \pm 15 \mathrm{Myr}$ & $(2)$ \\
& $32 \mathrm{Myr}$ & $(3)$ \\
& $32 \& 40 \mathrm{Myr}$ & $(4)$ \\
& $35-40 \mathrm{Myr}$ & $(5)$ \\
$\tau_{\mathrm{RSG}}$ & $45 \pm 5 \mathrm{Myr}$ & $(6)$ \\
$\log \left(\mathrm{M}_{\mathrm{dyn}} / \mathrm{M}_{\odot}\right)$ & $5.20 \pm 0.17$ & $(6)$ \\
$\log \left(\mathrm{M}_{\mathrm{phot}} / \mathrm{M}_{\odot}\right)$ & $4.6 \pm 0.2$ & $(7)$ \\
$v_{0}$ & $153.7 \pm 0.8 \mathrm{~km} \mathrm{~s}^{-1}$ & $(6)$ \\
$\sigma_{1 \mathrm{D}}$ & $3.20{ }_{-0.52}^{+0.69} \mathrm{~km} \mathrm{~s}^{-1}$ & $(6)$ \\
$f_{\mathrm{MS}}$ & $0.54 \pm 0.05$ & $(8)$ \\
$f_{\mathrm{RSG}}$ & $0.3 \pm 0.1$ & $(6)$ \\
\hline
\end{tabular}

Notes. (1) Grebel et al. (1994), (2) Chiosi et al. (1995), (3) Keller et al. (2000), (4) Milone et al. (2018), (5) Bodensteiner et al. (2019), (6) this study , (7) Mackey \& Gilmore (2003), (8) Li et al. (2017).

mass stars, which they argued should not be considered in the age analysis. Contamination from SMC-field stars is not likely to be significant for our sample in this respect, and we consider the exact distinction between RSG and massive-AGB stars, which are known to have an increasingly similar evolutionary path at lower metallicity (Doherty et al. 2010, 2015), unimportant for an analysis of the age of the cluster. We did consider the entire sample as genuine RSGs, however.

Comparing the age derived here with previous values in the literature, see Table 2, we find that the RSG age based on the current technique is older than that derived from more traditional approaches such as fitting the MSTO, or the mean positions of the RSG and BSG stars in the CMD, as discussed above.

We note that recent work by Milone et al. (2018) argued for two stellar populations in this cluster, one bi-modal population of $40 \mathrm{Myr}$, composed of a sub-population of stars rotating at close to critical rotational velocity, and a sub-population with low rotational velocity, and a second younger population of $32 \mathrm{Myr}$ with low rotational velocities. Figure 7 shows that this age spread cannot explain the observed spread in apparent ages of the RSGs in the cluster, which range from 15 to $50 \mathrm{Myr}$. However, spectroscopic studies of the brightest turn-off stars in NGC 330 consistently find them to be blue stragglers, which are B-type giants beyond the end of the classical main sequence (Grebel et al. 1996; Lennon et al. 2003). As Britavskiy et al. (2019) discussed, these may be the evolutionary ancestors of the bright RSGs.

\subsection{Evolutionary considerations}

Li et al. (2017) studied the extended main-sequence turn-off in four young massive clusters in the Magellanic Clouds (including NGC 330). Using HST photometry and synthetic CMDs, they estimated a main-sequence binary fraction of $f_{\mathrm{MS}}=0.54 \pm 0.05$ using the method of Milone et al.(2012) and taking into account all mass ratios assuming a flat mass-ratio distribution. This is significantly larger than our estimated $f_{\mathrm{RSG}}$, as expected. However, we caution that these estimates used very different approaches and therefore have very different biases. A more appropriate comparison would be with a binary fraction estimated using RV variations.

The spread of luminosities among the RSG population is potentially an indicator of post-interaction binary products, with a larger spread indicating greater 'contamination' from binarity.
The observed luminosity spread in NGC 330 is characteristic of those observed in other young massive clusters in the Galaxy and LMC and SMC (e.g. Patrick et al. 2016). Following the arguments in Britavskiy et al. (2019), we assumed that the relative luminosity range of a single RSG in a coeval cluster is $\sim 5 \%$ and therefore considered all RSGs with luminosities above $\log \mathrm{L} / \mathrm{L}_{\odot}=4.2$ to be red stragglers. This resulte in a red-straggler fraction in NGC 330 of 50\%, in excellent agreement with the results of Britavskiy et al. (2019). A more quantitative comparison is beyond the scope of this study, but if the spread of luminosities of RSGs can be attributed to binary post-interaction products, this would suggest similar binary fractions within young massive clusters.

\section{Conclusion}

We have investigated the multiplicity properties of the RSG population of NGC 330 using archival multi-epoch HARPS data supplemented with multi-epoch MUSE observations. Precise RVs were estimated for our targets with a slicing approach that is specifically designed to provide an unbiased estimate of the atmospheric RV, as presented by P19. Based on the stability and high resolution of the HARPS spectrograph, the estimated precision on the RVs for our RSGs is as small as $60 \mathrm{~m} \mathrm{~s}^{-1}$.

The line-of-sight velocity and its associated dispersion were estimated for the cluster, superseding past estimates that were limited by observational precision (Feast \& Black 1980). Given their apparent lack of RV variability, we argue that RSGs are effective kinematic tracers of young massive clusters. For a larger observational sample, we combined our estimates for the RSGs with RVs for nearby OBA-type massive stars, and the resulting velocity dispersion $\left(\sigma_{1 \mathrm{D}}=3.20_{-0.52}^{+0.69} \mathrm{~km} \mathrm{~s}^{-1}\right)$ is in good agreement with the value estimated from the RSGs alone.

When we assumed virial equilibrium, the dynamical mass of the cluster was estimated as $\log \left(\mathrm{M}_{\mathrm{dyn}} / \mathrm{M}_{\odot}\right)=5.20 \pm 0.17$, in good agreement with previous dynamical mass estimates and photometric mass estimates from Mackey \& Gilmore (2003) and Bodensteiner et al. (2019), assuming a binary fraction of $25 \%$ using the relations of Gieles et al. (2010).

The multiplicity properties of the RSGs were investigated by searching for significant variability within the multi-epoch RVs for each RSG. By varying the criteria to detect significant variability, we attempted to distinguish between variations from atmospheric motions and genuine binarity. To estimate the intrinsic binary fraction of the RSG population, we simulated binary populations using an empirically defined distribution of systems from Moe \& Di Stefano (2017). For orbital periods in the range $2.3<\log \mathrm{P}$ [days] $<4.3$ and mass ratios above 0.1 , we estimate an intrinsic binary fraction of $f_{\mathrm{RSG}}=0.3 \pm 0.1$. We compile the physical properties of NGC 330 in Table 2 and show that the binary fraction of RSGs appears significantly smaller than that of main-sequence stars, which is expected assuming that binary interactions remove RSG binaries.

Based on a sample of 20 RSGs, selected through the Gaia DR2 astrometry (15 of which have RVs presented in the current study), the age of NGC 330 is estimated using the distribution of luminosities of the RSG population of NGC 330 following Britavskiy et al. (2019). This technique takes into account the affect of binarity on the RSG population, and we estimate the cluster age to be $\tau_{\mathrm{RSG}}=45 \pm 5 \mathrm{Myr}$. We note that the suggestion of Milone et al. (2018), that the main-sequence population of NGC 330 consists of $32 \mathrm{Myr}$ and $40 \mathrm{Myr}$ populations, cannot explain the spread in luminosities that is observed in the RSG population. Using the luminosity distribution, we estimate a red 
straggler fraction of $50 \%$, in excellent agreement with Britavskiy et al. (2019).

Acknowledgements. The authors would like to thank the anonymous referee for a careful review that improved the article. L. R. P and A. H acknowledge support from grant AYA2015- 68012-C2- 1-P from the Spanish Ministry of Economy and Competitiveness (MINECO). This research is partially supported by the Spanish Government under grants AYA2015-68012-C2-1/2-P and PGC2018-093741-B-C21/2 (MICIU/AEI/FEDER, UE). This publication makes use of data products from the Two Micron All Sky Survey, which is a joint project of the University of Massachusetts and the Infrared Processing and Analysis Center/California Institute of Technology, funded by the National Aeronautics and Space Administration and the National Science Foundation. This work has made use of data from the European Space Agency (ESA) mission Gaia (https://www . cosmos.esa.int/gaia), processed by the Gaia Data Processing and Analysis Consortium (DPAC, https://www.cosmos.esa.int/web/ gaia/dpac/consortium). Funding for the DPAC has been provided by national institutions, in particular the institutions participating in the Gaia Multilateral Agreement. We acknowledge support from the FWO-Odysseus program under project G0F8H6N. This project has further received funding from the European Research Council under European Union's Horizon 2020 research programme (grant agreement No 772225 - MULTIPLES). TOPCAT (Taylor 2005) was used to handle the Gaia data used in this publication. This research made use of Astropy ${ }^{4}$ a community-developed core Python package for Astronomy (Astropy Collaboration et al.2013 2018,

\section{References}

Allende Prieto, C. 2007, AJ, 134, 1843

Arp, B. H. 1959, AJ, 64, 254

Astropy Collaboration, Price-Whelan, A. M., Sipőcz, B. M., et al. 2018, AJ, 156, 123

Astropy Collaboration, Robitaille, T. P., Tollerud, E. J., et al. 2013, A\&A, 558, A33

Badenes, C., Mazzola, C., Thompson, T. A., et al. 2018, ApJ, 854, 147

Balona, L. A. 1992, MNRAS, 256, 425

Beasor, E. R., Davies, B., Smith, N., \& Bastian, N. 2019, MNRAS, 266

Bodensteiner, J., Sana, H., Mahy, L., et al. 2019, arXiv e-prints, arXiv: 1911.03477

Bressan, A., Marigo, P., Girardi, L., et al. 2012, MNRAS, 427, 127

Britavskiy, N., Lennon, D. J., Patrick, L. R., et al. 2019, A\&A, 624, A128

Brott, I., de Mink, S. E., Cantiello, M., et al. 2011, A\&A, 530, A115

Carney, B. W., Janes, K. A., \& Flower, P. J. 1985, AJ, 90, 1196

Chiavassa, A., Haubois, X., Young, J. S., et al. 2010, A\&A, 515, A12

Chiavassa, A., Pasquato, E., Jorissen, A., et al. 2011, A\&A, 528, A120

Chiosi, C., Vallenari, A., Bressan, A., Deng, L., \& Ortolani, S. 1995, A\&A, 293, 710

Cignoni, M., Sabbi, E., van der Marel, R. P., et al. 2016, ApJ, 833, 154

Clark, J. S., Negueruela, I., Davies, B., et al. 2009, A\&A, 498, 109

Cunha, K., Sellgren, K., Smith, V. V., et al. 2007, ApJ, 669, 1011

Davies, B. \& Beasor, E. R. 2018, MNRAS, 474, 2116

Davies, B., Figer, D. F., Kudritzki, R.-P., et al. 2007, ApJ, 671, 781

Davies, B., Figer, D. F., Law, C. J., et al. 2008, ApJ, 676, 1016

Davies, B., Kudritzki, R.-P., Plez, B., et al. 2013, ApJ, 767, 3

De Marco, O. \& Izzard, R. G. 2017, PASA, 34, e001

de Mink, S. E., Langer, N., Izzard, R. G., Sana, H., \& de Koter, A. 2013, ApJ, 764, 166

Dicenzo, B. \& Levesque, E. M. 2019, arXiv e-prints, arXiv:1902.01862

Doherty, C. L., Gil-Pons, P., Siess, L., Lattanzio, J. C., \& Lau, H. H. B. 2015, MNRAS, 446, 2599

Doherty, C. L., Siess, L., Lattanzio, J. C., \& Gil-Pons, P. 2010, MNRAS, 401, 1453

Dorda, R., Negueruela, I., González-Fernández, C., \& Marco, A. 2018, A\&A, 618, A137

Dotter, A. 2016, ApJS, 222, 8

Dumusque, X., Pepe, F., Lovis, C., et al. 2012, Nature, 491, 207

Dunstall, P. R., Dufton, P. L., Sana, H., et al. 2015, A\&A, 580, A93

Ekström, S., Georgy, C., Eggenberger, P., et al. 2012, A\&A, 537, A146

Eldridge, J. J., Izzard, R. G., \& Tout, C. A. 2008, MNRAS, 384, 1109

Elias, J. H., Frogel, J. A., \& Humphreys, R. M. 1985, ApJS, 57, 91

Evans, C. J., Lennon, D. J., Smartt, S. J., \& Trundle, C. 2006, A\&A, 456, 623

Evans, C. J., Taylor, W. D., Hénault-Brunet, V., et al. 2011, A\&A, 530, A108

Feast, M. W. 1972, MNRAS, 159, 113

Feast, M. W. \& Black, C. 1980, MNRAS, 191, 285

\footnotetext{
4 http://www.astropy.org
}

Foreman-Mackey, D., Hogg, D. W., Lang, D., \& Goodman, J. 2013, PASP, 125, 306

Gaia Collaboration, Helmi, A., van Leeuwen, F., et al. 2018, A\&A, 616, A12

Gazak, J. Z., Davies, B., Kudritzki, R., Bergemann, M., \& Plez, B. 2014, ApJ, 788,58

Gieles, M., Sana, H., \& Portegies Zwart, S. F. 2010, MNRAS, 402, 1750

Gonzalez, G. \& Wallerstein, G. 1999, AJ, 117, 2286

González-Fernández, C., Dorda, R., Negueruela, I., \& Marco, A. 2015, A\&A, 578, A3

Goodman, J. \& Weare, J. 2010, Comm. App. Math. Comp. Sci., 1, 65

Götberg, Y., de Mink, S. E., Groh, J. H., et al. 2018, A\&A, 615, A78

Graczyk, D., Pietrzyński, G., Thompson, I. B., et al. 2014, ApJ, 780, 59

Grebel, E. K., Richter, T., \& de Boer, K. S. 1992, A\&A, 254, L5

Grebel, E. K., Roberts, W. J., \& Brandner, W. 1996, A\&A, 311, 470

Grebel, E. K., Roberts, W. J., Brandner, W., \& Moneti, A. 1994, in AAS Meeting Abstracts, Vol. 185, 51.12

Hill, V. 1999, A\&A, 345, 430

Josselin, E. \& Plez, B. 2007, A\&A, 469, 671

Keller, S. C., Bessell, M. S., \& Da Costa, G. S. 2000, AJ, 119, 1748

Kiminki, D. C. \& Kobulnicky, H. A. 2012, ApJ, 751, 4

Kobulnicky, H. A., Kiminki, D. C., Lundquist, M. J., et al. 2014, The Astrophysical Journal Supplement Series, 213, 34

Kravchenko, K., Chiavassa, A., Van Eck, S., et al. 2019, arXiv e-prints, arXiv: 1910.04657

Lapenna, E., Origlia, L., Mucciarelli, A., et al. 2015, ApJ, 798, 23

Lennon, D. J., Dufton, P. L., \& Crowley, C. 2003, A\&A, 398, 455

Lennon, D. J., Dufton, P. L., Mazzali, P. A., Pasian, F., \& Marconi, G. 1996 A\&A, 314, 243

Levesque, E. M. 2017, Astrophysics of Red Supergiants

Levesque, E. M. \& Massey, P. 2012, AJ, 144, 2

Li, C., de Grijs, R., Deng, L., \& Milone, A. P. 2017, ApJ, 844, 119

Lindegren, L., Hernández, J., Bombrun, A., et al. 2018, A\&A, 616, A2

Mackey, A. D. \& Gilmore, G. F. 2003, MNRAS, 338, 85

Maoz, D., Badenes, C., \& Bickerton, S. J. 2012, ApJ, 751, 143

Martayan, C., Floquet, M., Hubert, A. M., et al. 2007, A\&A, 472, 577

Massey, P. \& Olsen, K. A. G. 2003, AJ, 126, 2867

Mayor, M., Pepe, F., Queloz, D., et al. 2003, The Messenger, 114, 20

McCrea, W. H. 1964, MNRAS, 128, 147

McLaughlin, D. E. \& van der Marel, R. P. 2005, ApJS, 161, 304

Milone, A. P., Marino, A. F., Di Criscienzo, M., et al. 2018, MNRAS, 477, 2640

Milone, A. P., Piotto, G., Bedin, L. R., et al. 2012, A\&A, 540, A16

Moe, M. \& Di Stefano, R. 2017, ApJS, 230, 15

Momany, Y., Vandame, B., Zaggia, S., et al. 2001, A\&A, 379, 436

Neugent, K. F., Levesque, E. M., \& Massey, P. 2018, AJ, 156, 225

Neugent, K. F., Levesque, E. M., Massey, P., \& Morrell, N. I. 2019, ApJ, 875, 124

Patrick, L. R., Evans, C. J., Davies, B., et al. 2017, MNRAS, 468, 492

Patrick, L. R., Evans, C. J., Davies, B., et al. 2015, ApJ, 803, 14

Patrick, L. R., Evans, C. J., Davies, B., et al. 2016, MNRAS, 458, 3968

Patrick, L. R., Lennon, D. J., Britavskiy, N., et al. 2019, A\&A, 624, A129, [P19]

Pepe, F., Ehrenreich, D., \& Meyer, M. R. 2014, Nature, 513, 358

Plez, B. 2012, Turbospectrum: Code for spectral synthesis

Podsiadlowski, P., Joss, P. C., \& Hsu, J. J. L. 1992, ApJ, 391, 246

Portegies Zwart, S. F., McMillan, S. L. W., \& Gieles, M. 2010, ARA\&A, 48, 431

Robertson, J. W. 1974, A\&AS, 15, 261

Sana, H., de Koter, A., de Mink, S. E., et al. 2013, A\&A, 550, A107

Sana, H., de Mink, S. E., de Koter, A., et al. 2012, Science, 337, 444

Sana, H., Gosset, E., \& Evans, C. J. 2009, MNRAS, 400, 1479

Sana, H., Le Bouquin, J. B., Lacour, S., et al. 2014, The Astrophysical Journal Supplement Series, 215, 15

Sanford, R. F. 1933, ApJ, 77, 110

Schneider, F. R. N., Izzard, R. G., de Mink, S. E., et al. 2014, ApJ, 780, 117

Schwarzschild, M. 1975, ApJ, 195, 137

Smartt, S. J. 2009, ARA\&A, 47, 63

Smartt, S. J. 2015, PASA, 32, e016

Smette, A., Sana, H., Noll, S., et al. 2015, A\&A, 576, A77

Smith, M. A., Patten, B. M., \& Goldberg, L. 1989, AJ, 98, 2233

Sonneborn, G., Altner, B., \& Kirshner, R. P. 1987, ApJ, 323, L35

Spencer Jones, H. 1928, MNRAS, 88, 660

Spite, F., Spite, M., \& Richtler, T. 1991, A\&A, 252, 557

Stothers, R. B. 2010, ApJ, 725, 1170

Tabernero, H. M., Dorda, R., Negueruela, I., \& Gonzá lez-Fernández, C. 2018, MNRAS, 476, 3106

Taylor, M. B. 2005, in Astronomical Society of the Pacific Conference Series, Vol. 347, Astronomical Data Analysis Software and Systems XIV, ed. P. Shopbell, M. Britton, \& R. Ebert, 29

Taylor, W. D., Evans, C. J., Simón-Díaz, S., et al. 2014, MNRAS, 442, 1483

Wright, K. O. 1977, JRASC, 71, 152 


\section{Appendix A: Multi-epoch radial velocities}

Table A.1 provides the complete list of estimated RVs for the RSGs considered in this paper as well as literature measurements.

Figure A.1 displays RV measurements for our targets alongside literature measurements spanning a baseline of more than 40 years. This figure highlights the excellent agreement between the RV measurements presented in this study with those published previously, and simultaneously highlights the lack of significant RV variability over this timescale. However, because it is difficult to determine the systematic errors that might be present in these previous results, we did not include these data in our quantitative determination of the binary fraction. Instead, we provide a brief qualitative discussion of these results here.

Feast \& Black (1980) published some of the earliest multiepoch RVs for the brightest stars in NGC 330, finding an unusually small fraction of variables that they interpreted as evidence for a small binary fraction in the cluster. Their measured values for several RSGs in the cluster show little dispersion per star, with an observational uncertainty per star of $\sim \pm 1-3 \mathrm{~km} \mathrm{~s}^{-1}$. There is an indication of bimodality in their results, however, that suggests some unresolved systematic uncertainty for some stars of $\sim 5 \mathrm{~km} \mathrm{~s}^{-1}$. Carney et al. (1985) measured RVs for many stars in the cluster with similar mean results as Feast \& Black (1980), but with uncertainties of $\sim 10 \mathrm{~km} \mathrm{~s}^{-1}$ for each star. The highresolution echelle observations of Spite et al.(1991), Hill (1999) and Gonzalez \& Wallerstein (1999) have quoted uncertainties of $1 \mathrm{~km} / \mathrm{s}$, derived from cross correlation with standards, and compare extremely well with the HARPS results presented here (but for some observations, only approximate dates are given). These echelle observations are remarkably constant, within the small uncertainties, and are all consistent with the systemic RV of the cluster. The overall picture therefore is that the RSG RVs discussed in this paper are remarkably constant over a time span of $\sim 40$ years.

\section{Appendix B: Literature IDs}

Compiling the archival data used in this study, we realised that owing to the dense cluster environment, identifying the targets in different literature studies is often difficult. To aid future studies, here we cross-match the identifications of several well-known studies of NGC 330 with Gaia DR2 identifications. Table B.1 lists the results including Gaia astrometry for the sources. 
Table A.1. RV estimates at each epoch for RSGs in the HARPS and MUSE samples.

\begin{tabular}{|c|c|c|c|c|}
\hline ID & JD & $\mathrm{RV}\left(\mathrm{km} \mathrm{s}^{-1}\right)$ & $\sigma_{\mathrm{RV}}\left(\mathrm{km} \mathrm{s}^{-1}\right)$ & Spectrograph/Reference \\
\hline A3 & 2443462.5 & 155.0 & 6 & 1980MNRAS.191..285F \\
\hline A3 & 2450728.5 & 150.5 & 1 & 1999AJ....117.2286G \\
\hline A3 & 2448544.5 & 155.4 & 1 & 1999A\&A...345..430H \\
\hline A3 & 2448544.5 & 155.1 & 1 & 1999A\&A...345..430H \\
\hline A3 & 2455087.7 & 150.77 & 0.19 & HARPS \\
\hline A3 & 2455088.6 & 151.39 & 0.17 & HARPS \\
\hline A3 & 2455100.7 & 151.10 & 0.24 & HARPS \\
\hline A3 & 2455120.7 & 151.37 & 0.15 & HARPS \\
\hline A3 & 2455144.5 & 150.99 & 0.15 & HARPS \\
\hline A3 & 2455145.6 & 150.70 & 0.30 & HARPS \\
\hline A3 & 2455185.6 & 150.52 & 0.20 & HARPS \\
\hline A3 & 2455430.7 & 150.89 & 0.17 & HARPS \\
\hline A3 & 2455432.6 & 151.00 & 0.34 & HARPS \\
\hline A3 & 2455447.6 & 152.05 & 0.09 & HARPS \\
\hline A3 & 2455467.6 & 151.15 & 0.17 & HARPS \\
\hline A3 & 2455470.5 & 150.54 & 0.15 & HARPS \\
\hline A3 & 2455477.5 & 151.82 & 0.08 & HARPS \\
\hline A3 & 2455479.6 & 151.89 & 0.20 & HARPS \\
\hline A3 & 2455502.5 & 151.43 & 0.16 & HARPS \\
\hline A3 & 2455535.6 & 151.80 & 0.16 & HARPS \\
\hline$\ldots$ & $\ldots$ & $\ldots$ & $\ldots$ & $\ldots$ \\
\hline
\end{tabular}

Notes. A full version of this table is available electronically. The first 20 lines are shown as a sample.

Table B.1. Identifications for RSGs in NGC 330 within a 2'5 radius of the cluster centre.

\begin{tabular}{lccccc}
\hline \hline ID $_{R 74}$ & ID $_{\text {Arp59 }}$ & ID $_{\text {Bal92 }}$ & ID $_{\text {GaiaDR2 }}$ & RA & DEC \\
\hline A3 & ARP26 & BAL690 & 4688993681762966400 & $00: 56: 21.6996$ & $-72: 27: 35.8594$ \\
A6 & ARP43 & BAL450 & 4688993647403231232 & $00: 56: 13.8954$ & $-72: 27: 32.4019$ \\
A7 & ARP41 & BAL475 & 4688993647403173504 & $00: 56: 14.5617$ & $-72: 27: 42.4507$ \\
A9 & ARP42 & BAL423 & 4688993647403241088 & $00: 56: 12.8613$ & $-72: 27: 46.6309$ \\
A14 & ARP17 & BAL623 & 4688993471264541056 & $00: 56: 19.0367$ & $-72: 28: 08.2365$ \\
A27 & ARP32 & BAL561 & 4688993681762883584 & $00: 56: 17.0829$ & $-72: 27: 35.0108$ \\
A42 & ARP21 & BAL645 & 4688993681762909440 & $00: 56: 20.1423$ & $-72: 27: 47.0941$ \\
A45 & ARP38 & BAL618 & 4688993681762919040 & $00: 56: 18.8279$ & $-72: 27: 47.4984$ \\
A46 & ARP37 & BAL595 & 4688993677426863872 & $00: 56: 18.0162$ & $-72: 27: 49.8524$ \\
A52 & ARP36 & - & 4688993681703883648 & $00: 56: 17.3709$ & $-72: 27: 52.5296$ \\
A57 & ARP19 & BAL619 & 4688993681762914304 & $00: 56: 19.4722$ & $-72: 27: 53.2203$ \\
B15 & ARP29 & BAL523 & 4688993750482336640 & $00: 56: 16.5574$ & $-72: 27: 04.6490$ \\
B31 & ARP02 & BAL317 & 4688993643141502336 & $00: 56: 09.4247$ & $-72: 28: 09.4895$ \\
B40 & ARP10 & BAL801 & 4688993475604563584 & $00: 56: 25.3298$ & $-72: 28: 18.2008$ \\
B42 & ARP12 & BAL878 & 4688993471342994048 & $00: 56: 27.3354$ & $-72: 28: 06.4611$ \\
B10 & ARP III-224 & - & 4688999557280890496 & $00: 56: 36.3522$ & $-72: 26: 46.4698$ \\
B19 & ARP III-205 & - & 4688994368957731584 & $00: 55: 55.7677$ & $-72: 27: 09.8270$ \\
B20 & ARP III-203 & BAL90 & 4688993716122719872 & $00: 56: 00.9736$ & $-72: 27: 39.6590$ \\
- & ARP III-210 & - & 4688994472036827392 & $00: 56: 10.9903$ & $-72: 26: 26.0654$ \\
- & ARP III-214 & - & 4688994575116023808 & $00: 56: 14.7713$ & $-72: 25: 20.7020$ \\
\hline
\end{tabular}

Notes. Literature identification from Robertson (1974) and Balona (1992). 

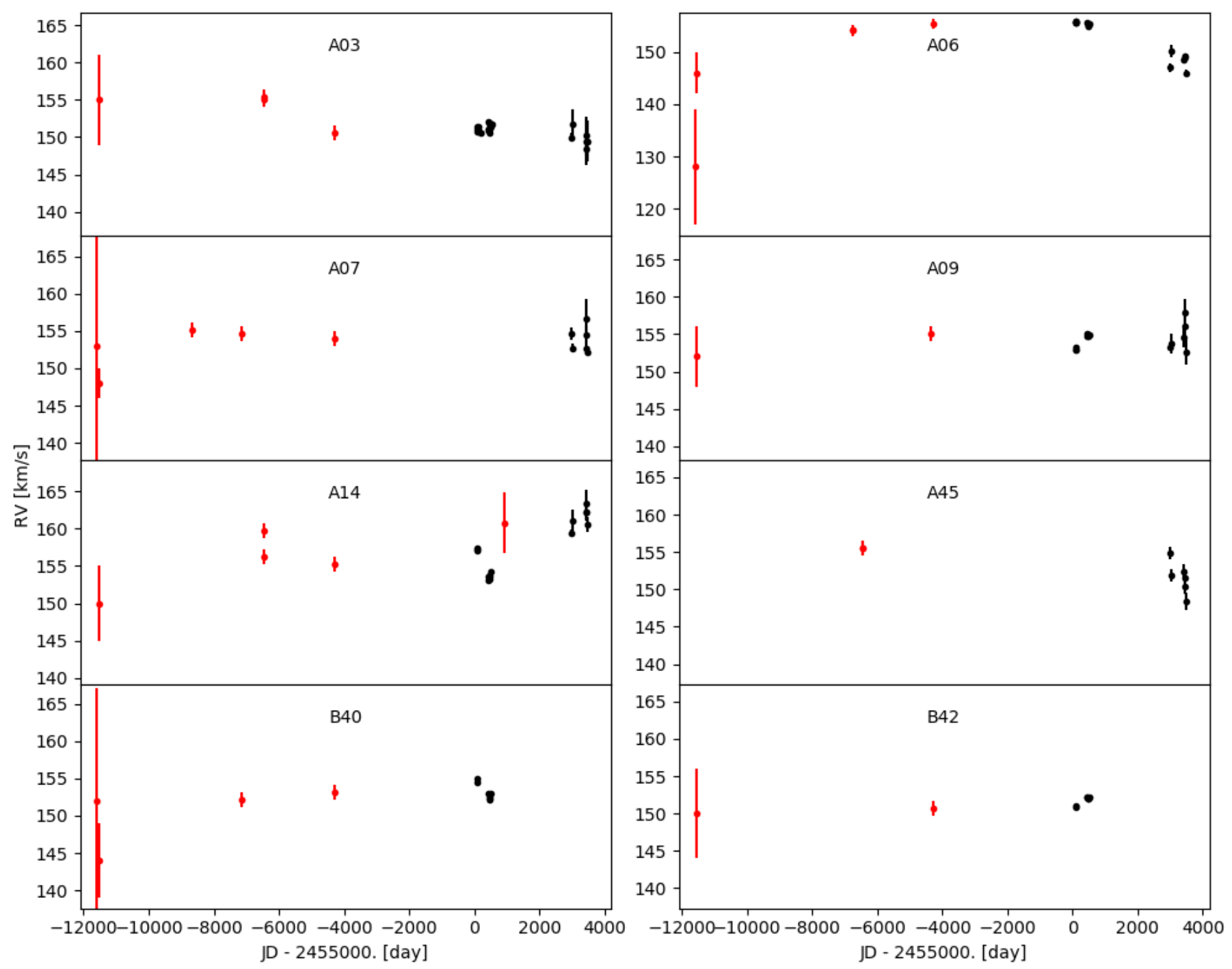

Fig. A.1. RV estimates for all targets with literature measurements, shown on the same relative scale to demonstrate the observed differences in $\mathrm{RV}$ variations (except for Rob74 B31, which will be discussed in detail in an upcoming publication). Red data points indicate RV estimates from literature studies. In general, the agreement with the literature measurements is excellent. 